\title{
Editorial
}

\section{A nuestros lectores}

Este año que acaba ha sido realmente muy complicado. La pandemia del COVID-19 ha afectado la vida diaria de todos de una manera sin precedentes. A lo largo de estos tiempos difíciles, hemos tenido que readaptar nuestras costumbres, relaciones, viajes, trabajo, a una nueva realidad que nadie se esperaba e incluso imaginaba. Nuestra casa se ha transformado y ahora forma parte de nuestro entorno de trabajo impartiendo clases online, videoconferencias, reuniones virtuales, etc. Hemos descubierto multitud de programas tipo Zoom, Teams, Webex, Meet, Skype, para conectarnos entre nosotros y mantener la actividad laboral.

La actividad docente y de investigación se ha reanudado de forma paulatina y en general todo funciona razonablemente; esperemos, que con la vacuna, el próximo año sea más "normal". Desde la revista Óptica Pura y Aplicada hemos intentado mantener la actividad pese a todas estas dificultades. Hemos sufrido dos hechos significativos, por un lado, la disminución del número de artículos recibidos y por el otro, el retraso de las publicaciones. La disminución de los trabajos nos ha obligado a plantear nuevas estrategias para atraer nuevos trabajos desde artículos de investigación, tutoriales, revisión (review), docentes, .... hasta números temáticos o especiales de congresos o en colaboración con otras sociedades afines. Por ejemplo, mediante el convenio de colaboración de SEDOPTICA con Red Colombiana de Óptica-RCO (ver Newsletter) se ha planteado la realización de un número especial sobre la Óptica en Colombia.

En general, el problema del retraso de la publicación viene dado por la falta de revisores y cada vez es más difícil encontrar personas dispuestas a dedicar su tiempo a la revisión. Intentamos solventar el problema con una base de datos de antiguos revisores, junto con los sugeridos por los autores al mandar el artículo. Aún así, no es fácil y tenemos que echar mano de colegas para tener el trabajo revisado en un tiempo razonable. Somos conscientes de que los autores y revisores están haciendo todo lo posible dadas las actuales circunstancias. En este sentido es justo reconocer públicamente esta actividad fundamental para la revista y en el primer número de OPA de 2021, se publicará una lista de los revisores que hemos tenido durante el 2020.

Por otro lado, espero que el nuevo año nos traiga una nueva plataforma para la gestión de los trabajos mandados a OPA. La actual plataforma es de mucha utilidad y ha permitido una gestión ordenada y sistemática de la revista, pero no es perfecta y se puede mejorar. Se está acabando de desarrollar y probar una nueva que permitirá ser más ágiles y eficientes en la gestión de los artículos y en parte, quizás disminuir el tiempo de publicación.

Ahora más que nunca, es importante mencionar que todas las sugerencias para mejorar son bienvenidas y que durante estos tiempos difíciles, nuestro objetivo ha sido y será apoyar a comunidad científica y docente de la mejor manera posible. Por esto quiero acabar con un agradecimiento a nuestros lectores, autores y revisores por continuar con nosotros y su apoyo a la revista Óptica Pura y Aplicada.

Saludos y feliz año nuevo 2021.

Tarragona, Diciembre de 2020

Lluís F. Marsal,

Editor de Óptica Pura y Aplicada

DOI: http://dx.doi.org/10.7149/OPA.53.4.i 